\title{
The Influence of False Cardiac Feedback on Autonomic Markers of Arousal
}

\author{
Marie Kurrátková, Eva Kozáková, \\ Radek Mareček, Robert Roman \\ Central European Institute of Technology, \\ Masaryk University, Kamenice 5 Brno, \\ Czech Republic
}

\author{
Ondřej Bečev, Lenka Sakálošová, \\ Milan Brázdil \\ Central European Institute of Technology, \\ Masaryk University, Kamenice 5 Brno, \\ Czech Republic \\ Faculty of Medicine, Masaryk University, \\ Kamenice 5, Brno, Czech Republic
}

\author{
Bartosz Majchrowicz \\ Consciousness Lab, Institute of Psychology, \\ Jagiellonian University, Kraków, Poland
}

\begin{abstract}
Interoception plays a key role in experiencing emotions, as it provides information about bodily arousal. Cardiac feedback manipulation proved to be a useful tool for exploring interoceptive processes. However, previous research showed inconclusive results on the influence of synchronous and false cardiac feedback on autonomic arousal. In the present study, we measured the influence of accelerated false cardiac feedback on participants' heart rate, respiratory rate and heart rate variability. Furthermore, we were interested in the modulatory effect of interoceptive accuracy measured by the Schandry task. Participants' ECG and respiratory rate were recorded during experimental task involving presentation of synchronous and false auditory cardiac feedback. Contrary to our assumption, false cardiac feedback elicited deceleration of heart rate and reduction of heart rate variability. Interoceptive accuracy had no effect on either variable. According to participant's reports, cardiac and the respiratory deceleration may come from down-regulatory responses to the distorted feedback.
\end{abstract}

Key words: false cardiac feedback, interoception, heart rate, respiratory rate, autonomic arousal

\section{Introduction}

Our organism continuously monitors its internal state to ensure optimal energy expendi- ture in order to retain homeostatic balance. The ability to perceive the internal state and processes of one's body, such as heart rate (HR), temperature, oxygen level, etc., is labelled interoception (Barrett \& Simmons, 2015; Seth,

\section{Acknowledgements}

This work was supported by the project CZ.02.1.01/0.0/0.0/16_013/0001775 "Modernization and support of research activities of the national infrastructure for biological and medical imaging Czech-BioImaging" funded by OP RDE. We are grateful to Eva Korit'áková for her invaluable comments that helped improve the paper. We acknowledge the core facility MAFIL of CEITEC, supported by the Czech-BioImaging large RI project (LM2015062 funded by MEYS CR), for their support with obtaining the scientific data presented in this paper.

Correspondence concerning this article should be addressed to Mgr. Marie Kuřátková, Central European Institute of Technology, Masaryk University, Kamenice 5 Brno, Czech Republic. E-mail: marie.kuratkova@gmail.com

Received June 22, 2018 
2013; Murphy, Brewer, Catmur, \& Bird, 2017). Subjects with good interoceptive ability are better in perceiving their physiological condition and are more aware of their physiological changes (Garfinkel, Seth, Barrett, Suzuki, \& Critchley, 2015). Interoception has been modeled as having three dimensions (Garfinkel et al., 2015, p. 66). One of the dimensions is interoceptive accuracy, which represents the objective accuracy in detecting bodily signals and it can be measured with objective methods, such as the Schandry task (Schandry, 1981).

Interoception plays an important role in experiencing emotions, since it provides information about bodily arousal (Wiens, 2005). For example, Wiens, Mezzacappa, and Katkin (2000) found that "good detectors" of their own heartbeat reported higher intensity of emotions than "poor detectors", while there was no difference in experience of emotional valence between both groups. The importance of interoception for adequate functioning is further illustrated by differences in interoceptive ability between patient groups. Patients with anorexia nervosa (Pollatos, Kurz et al., 2008), anxiety, social anxiety and panic disorder exhibit differences in interoceptive capacities (Stevens et al., 2011; Domschke, Stevens, Pfleiderer, \& Gerlach, 2010). It has also been put forward that disturbances in adequate interoception are linked to depersonalization and dissociative disorders (Seth, Suzuki, \& Critchley, 2012). Some studies suggest that some patients are better in the capacity to detect interoceptive signals (Stevens et al., 2011; Van der Does, Antony, Ehlers, \& Barsky, 2000), while others place the difference on the metacognitive level of beliefs about such signals (Yoris et al., 2015).

The interoceptive representation of internal states can be experimentally manipulated to study how the representation of the physical state affects participants' performance in experimental tasks, their subjective arousal and physiological reaction (Kleint, Wittchen, \& Lueken,
2015; Wild, Clark, Ehlers, \& McManus, 2008). Interestingly, the effect of such manipulation on physiological reactions varies according to interoceptive capacities or psychiatric conditions. One way to manipulate the representation of the physiological state is through distorted external feedback, usually called false cardiac feedback (FCF). During false cardiac feedback, a person is exposed to a visual or an auditory cardiac feedback that is artificially accelerated, decelerated or shifted (also called asynchronous) (Gray, Harrison, Wiens, \& Critchley, 2007). The person exposed to this feedback is not informed about the experimental manipulation.

Previous studies provided conflicting evidence on the influence of FCF on experienced arousal, measured by skin conductance and HR. Makkar and Grisham (2013) reported no significant effect of accelerated and decelerated FCF on the actual HR in groups of healthy low and high socially anxious participants. However, their results demonstrated that accelerated false feedback negatively affects mood, performance appraisals, and self-related cognition. Similarly, healthy participants in Kleint et al. (2015) reported a higher subjective arousal after the accelerated FCF conditions, although objective skin conductance did not differ. Conversely, Story and Craske (2008) reported that HR increased in response to increasing FCF in two extreme groups of healthy participants with and without elevated anxiety sensitivity. There was no significant difference in skin conductance. In sum, these results suggest that while the subject's reaction to FCF may not be recorded by physiological measures, they may nevertheless experience reportable subjective effects.

The extent of arousal during FCF could also be affected by other psychological factors, e.g. interoceptive accuracy. Higher interoceptive accuracy seems to correlate with one's ability to regulate arousal related to the emotional stimuli. Füstös, Gramann, Herbert, and Pollatos 
(2012) showed that the more aware a person was of bodily processes (higher interoceptive accuracy), the more successful they were in down-regulating the emotional response to negative affect evoked by emotionally stimulating pictures. Kever, Pollatos, Vermeulen, and Grynberg (2015) found a similar link between interoceptive accuracy and emotion regulation - better detection of bodily signals facilitated the selection and implementation of emotion regulation strategies. Bogaerts, Notebaert, Van Diest, Devriese, De Peuter, and Van den Bergh (2005) found that participants with high negative affectivity have a reduced accuracy in the perception of respiratory symptoms. These results support the conclusion that in healthy subjects, the higher interoceptive awareness positively correlates with participants' ability to down-regulate their emotional response to negative affect. Further investigation of interoceptive accuracy can clarify how it affects regulation of physiological reaction during emotional response and could reveal how lower interoceptive ability in different groups of psychiatric patients impacts their emotional regulation.

\section{The Present Study}

The purpose of this study is to expand on the results of Kleint et al. (2015) and to test whether the reported and experienced change in arousal in response to false cardiac feedback is traceable in other physiological measures, such as the HR or respiration.

We recorded heart rate $(\mathrm{HR})$ and respiratory rate (ResR) during two conditions: accelerated false cardiac feedback (FCF) and synchronous cardiac feedback (SCF). Since a decrease in heart rate variability (HRV) can be used as a measure of acute stress (e.g., Rieber et al., 2009; Dimitriev, Dimitriev, Karpenko, \& Saperova, 2008), we also analyzed HRV as an indicator of experienced stress throughout the experiment.
We used Schandry Mental Tracking Task to assess whether interoceptive accuracy influences the change in ResR, HR and HRV during FCF. As previously mentioned, studies suggest that higher interoceptive accuracy correlates with the ability to down-regulate emotional responses to negative affect (Füstös et al., 2012; Kever et al., 2015). The question is to what extent this down-regulation will be observable in HR, ResR and HRV and if it is possible that participants with higher interoceptive accuracy can, due to suggested down-regulation of negative affect, experience milder physiological response to false cardiac feedback.

\section{Hypotheses}

Three main hypotheses were investigated. It was hypothesized that 1) the heart rate (HR) and respiratory rate (ResR) will be higher in FCF blocks compared to SCF blocks; 2) participants' heart rate variability (HRV) will be smaller in FCF blocks than in SCF blocks; 3) participants with higher interoceptive accuracy scores will react to FCF with smaller changes in HR, ResR and HRV than participants with lower interoceptive accuracy scores.

\section{Methods}

\section{Participants}

In total, 36 subjects, mostly university students, were recruited via social network groups. Only healthy, right-handed subjects not suffering from any cardiac disease, neurological or psychiatric disorder were accepted to participate in the experiment. Due to technical issues (high number of artefacts in the HR recording), the data obtained from 9 participants had to be excluded from the analyses. The final sample for analyses included 27 subjects (10 males, mean age $=25.1, S D=5.41)$. All subjects provided written informed consent prior to the ex- 
periment and were informed about the purpose of the study. This experiment was approved by the ethical committee of Masaryk University.

\section{Experimental Design}

The experiment had a $2 \times 2$ within-subject design with cardiac feedback condition (FCF/SCF) and IB condition (active/passive). During this experiment, participants performed the Intentional Binding (IB) task while the auditory cardiac feedback stimulation in the form of beeps was played through loudspeakers. Participants' electrocardiography (ECG), skin conductance and respiratory rate (ResR) were measured during this task. Schandry Mental Tracking Task (Schandry, 1981) was used for measuring the participants' interoceptive accuracy. A short demographic questionnaire was also included (age, education, etc.). In this study, only the physiological data (ECG, RR) from the IB task will be presented. Due to technical issues during the data acquisition and poor data quality, skin conductance data were excluded from the subsequent analyses.

\section{Procedure}

Prior to the experiment, the participants were asked to fill in an online demographic questionnaire. Upon their arrival, they were informed about how the experimental procedure would proceed. They were told that the experiment was aimed at studying the brain's reactions to the auditory feedback of their heart activity. Participants were not informed that the cardiac feedback may be manipulated.

Recording took place in a shielded EEG Lab. Electrodes for ECG and skin conductance and respiratory belt were placed on participants prior to both tasks. Electrocardiogram (ECG) and respiratory curve were recorded with a BrainProducts BrainAmp ExG MR amplifier with an AUX box (BrainProducts, GmbH, Ger- many) with a sampling frequency of $5 \mathrm{kHz}$ per channel. The ECG signal was recorded via 2 electrodes placed on participants' chest - one on the lower sternum level, the other under the midpoint of the left armpit. For the purpose of the cardiac feedback, the acquired ECG was processed in real time via MATLAB (MathWorks) in blocks of $20 \mathrm{~ms}$ ( 100 samples) of the ECG signal. The signal variance computed for each block separately constituted latent signal with the sample rate of $50 \mathrm{~Hz}$ that contained peaks corresponding to heart beats. A short time before the IB task we manually set a threshold for heart beat detection that was controlled and adjusted when needed during the whole experiment. The respiratory curve was recorded via a pneumatic respiration belt sensor placed around the participants' chest.

First, participants undertook Schandry Mental Tracking Task which lasted approximately 10 minutes. The participants had to count their heartbeats during three different time periods $(35,45$, and $25 \mathrm{~s})$ only by concentrating on bodily feelings that can be associated with their heart. They were not allowed to take their pulse or to use any other means that could possibly help them in their estimation.

Second, they undertook the IB task, which lasted approximately 30 minutes and consisted of 4 blocks - 2 active and 2 passive (counterbalanced APAP/PAPA). Each section consisted of 12 trials. The trial was initiated by fixation cross screen. In following $2.6 \mathrm{sec}$ long window, the action was performed either voluntarily by a participant (Active condition) or computer (Passive condition). The passive condition differed from the active by means of the action execution. In the active condition, action had a form of voluntary button press on a keyboard, while in the passive condition, participants remained passive and the computer-triggered action had a form of auditory click-sound. After a short delay $(600,900$ or $1200 \mathrm{~ms})$ an effect (sound) was presented followed by the grey 
reporting screen. In this final part, participants indicated perceived time delay between the action and effect by an interval reproduction method - holding the key for a duration corresponding to the perceived action-effect interval.

The participants listened to auditory cardiac feedback through loudspeakers during the whole experiment (a 100ms $-440 \mathrm{~Hz}$ sine wave). The feedback was presented in two different conditions - false cardiac feedback (FCF) and synchronous cardiac feedback (SCF). SCF corresponded to the participants' heartbeat, i.e. the beep was played immediately after cardiac $\mathrm{R}$-wave detection. In the FCF, the beeps representing heart beats were accelerated. Inter-beep intervals were shortened to $70 \%$ of the interbeat intervals. These two types of cardiac feedback were played in alternating, twenty-sevensecond long blocks, 48 blocks in total.

At the end of the experimental session, participants were debriefed and provided with the explanation of the real purpose of the experiment.

\section{Statistical Analysis}

The ECG was pre-processed in Brain Vision Analyzer 2.0 (BVA, Brain Products). We used a semi-automatic detection of R-waves implemented in BVA. By comparing a timing of realtime detected heart beats to a timing of the offline detected R-waves, we were able to check the accuracy of real-time detection and feedback itself. Whenever we found more than a single error of real-time detection during the 27-s block of any type of cardiac feedback, the block was discarded from the ensuing analyses. The error was defined as a timing difference bigger than $100 \mathrm{~ms}$. The median of discarded blocks was 2 out of 48 (lower quartile $=0$; upper quartile $=8$ ).

For the statistical analysis, a mean value of the HR in beats per minute (bpm) was calculated for each condition. The standard devia- tion of RR intervals (SDNN) and mean value of root mean square of successive RR interval differences (RMSSD) (Berntson, Lozano, \& Chen, 2005) were used as indicators of heart rate variability in each condition. SignalPlant (Nejedly \& Virgala, 2016) was used for the analysis of the respiratory curve recordings. Mean values of the ResR in breaths per minute (bpm) were calculated for each condition.

The interoceptive accuracy (IA) score was calculated as the mean score across three heartbeat perception intervals using the following transformation based on Pollatos, TrautMattausch et al. (2007, p. 935):

$$
\frac{1}{3} \Sigma\left(1-\frac{\mid \text { recorded heartbeats }- \text { counted heartbeats } \mid}{\text { recorded heartbeats }}\right)
$$

The interoceptive accuracy score (IA score) can vary from 0 to 1 . High scores indicate strong interoceptive accuracy, i.e. only a small difference between the counted and the recorded heartbeats. The IA score was centered to the mean.

Due to the experimental design, $2 \times 2$ ANCOVA for repeated measures was used for analysis of heart rate, with feedback condition (false - synchronous) and IB condition (passive - active) and the IA score as a covariate. Dependent $t$-tests were used for heart rate post hoc analysis. In total, five tests were performed with subsequent pairs: FCF Passive vs. SCF Passive; FCF Active vs. SCF Active; FCF Passive vs. FCF Active; SCF Passive vs. SCF Active; difference in passive condition vs. difference in active condition. Bonferroni correction was used for $p$-value adjustment.

For the analysis of SDNN, 2x2 ANCOVA for repeated measures was also used. As a post hoc analysis, the dependent $t$-tests were used for SDNN. The same pairs and correction as in the analysis of HR were used. Bonferroni correction was also used for $t$-test $p$-values adjustment.

Due to the non-normal distribution of RMSSD and respiratory rate data, logarithmic transfor- 
mation was used for normalization. The data were non-normally distributed even after logarithmic transformation. For this reason, Wilcoxon signed-ranks tests were used for the analysis. The same pairs as in post hoc tests were used and Bonferroni correction was also used.

\section{Results}

Heart rate in false blocks compared to synchronous blocks differed significantly $[F(1,25)$ $\left.=18.19, p<0.001, \eta_{p}^{2}=0.42\right]$. Also, the interaction between feedback and IB condition was significant $\left[F(1,25)=6.30, p=0.019, \mathrm{\eta}_{p}^{2}=0.20\right]$. The HR in the IB condition differed more during the synchronous feedback blocks (Table 1, Figure 1, Figure 2) than during the false feedback blocks. There were no other significant results between other conditions and no significant interaction of any condition with IA score (Table 2).
The dependent $t$-tests showed that the FCF in the passive condition (FCF Passive vs. SCF Passive) did elicit a significant change in HR $[t(26)=-4.95, p<0.001$, Cohen's $d=-0.95]$. The HR was slower during the FCF blocks (mean = $74.48, S D=10.97)$ compared to the SCF blocks $($ mean $=75.35, S D=11.16)$ (Table 1, Figure 1, Figure 2). There were no other significant results in paired $t$-tests (Table 2).

Feedback condition had a significant effect on $\operatorname{SDNN}\left[F(1,25)=12.36, p=0.002, \eta_{p}^{2}=0.33\right]$. There were no other significant results between other conditions and no significant interaction of any condition with IA score (Table 2).

Dependent $t$-tests showed that the FCF in the passive condition (FCF Passive vs. SCF Passive) did elicit a significant change in SDNN $[t(26)=-2.80, p=0.05$, Cohen's $d=-0.54]$. The SDNN was lower during the FCF blocks (mean $=52.00, S D=15.98)$ compared to the SCF blocks $($ mean $=56.74, S D=15.99)($ Table 1, Figure 1 , Figure 2). The SDNN during the FCF blocks in

Table 1 Means and standard deviations of the heart rate (beats per minute), the respiratory rate (breaths per minute), SDNN (ms) and RMSSD (ms) for the Cardiac Feedback and the IB conditions in the IB task

\begin{tabular}{lcccc}
\hline & \multicolumn{2}{c}{ Heart rate (bpm) } & \multicolumn{2}{c}{ SDNN (ms) } \\
& Mean & \multicolumn{1}{c}{$S D$} & Mean & $S D$ \\
\hline SCF Active & 74.90 & 11.31 & 54.86 & 14.72 \\
SCF Passive & 75.35 & 11.16 & 56.74 & 15.99 \\
FCF Active & 74.51 & 11.03 & 49.79 & 14.52 \\
FCF Passive & 74.48 & 10.97 & 52.00 & 15.98 \\
Difference FCF A - SCF A & -0.39 & 0.89 & -5.07 & 8.41 \\
Difference FCF P - SCF P & -0.86 & 0.90 & -4.74 & 8.80 \\
\hline & \multicolumn{3}{c}{ Respiratory rate (bpm) } \\
\hline & RMSSD (ms) & Mean & $S D$ \\
\hline SCF Active & Mean & 13.65 & 17.27 & 2.31 \\
SCF Passive & 33.83 & 12.98 & 17,37 & 2.08 \\
FCF Active & 34.33 & 13.91 & 17.49 & 2.23 \\
FCF Passive & 34.26 & 15.02 & 17.15 & 2.42 \\
Difference FCF A - SCF A & 35.93 & 3.62 & 0.12 & -1.04 \\
Difference FCF P - SCF P & 0.42 & 6.45 & -0.13 & -1.95 \\
\hline
\end{tabular}



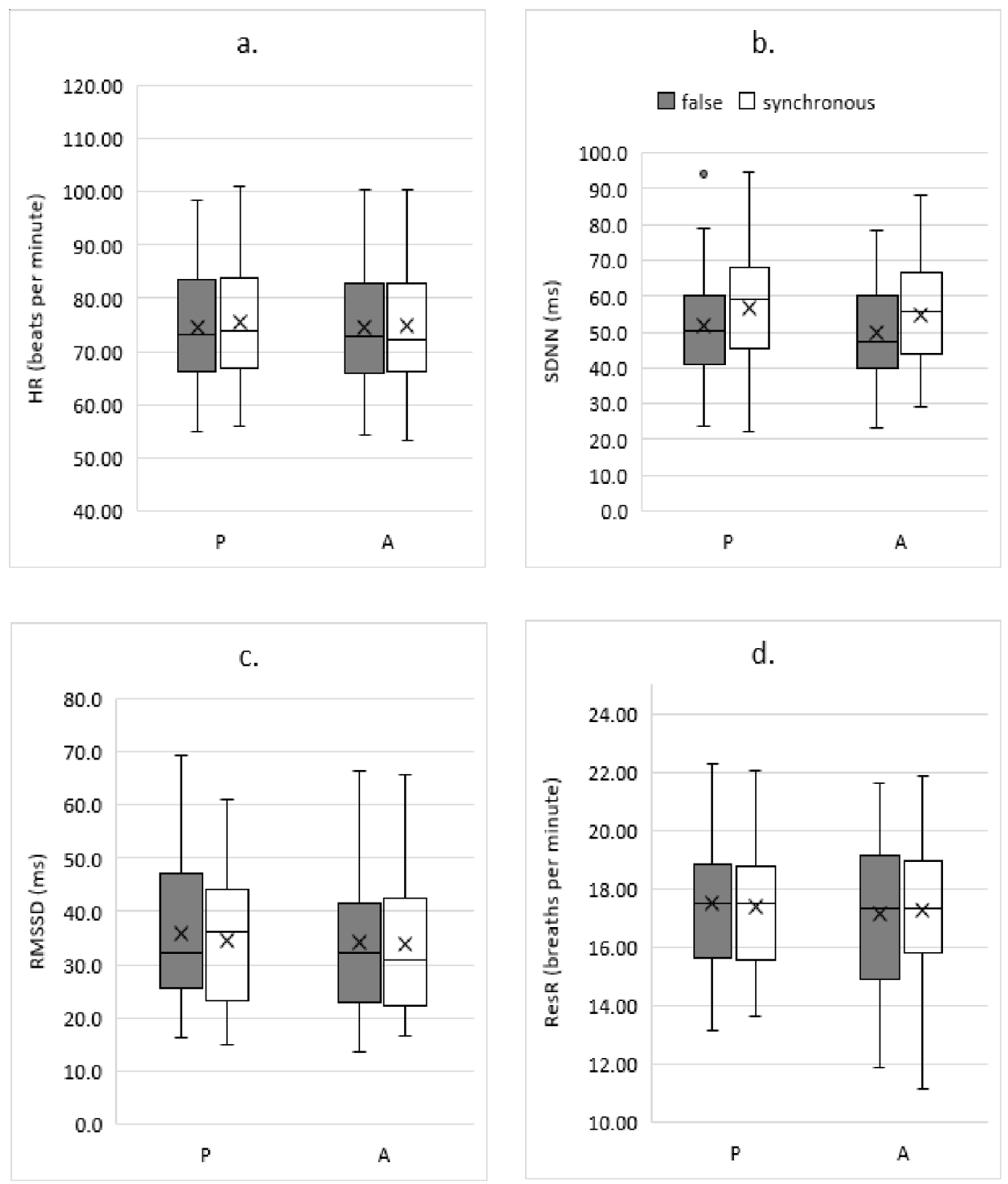

Figure 1 Mean (cross) and median a) heart rate (beats per minute), b) SDNN (ms), c) RMSSD (ms), d) respiratory rate (breaths per minute) for the Feedback and the IB condition in the IB task (box - first and third quartile, whiskers - minimum and maximum value). 




Figure 2 Mean and 95\% confidential interval a) heart rate (beats per minute), b) SDNN (ms), c) RMSSD $(\mathrm{ms}), \mathrm{d})$ respiratory rate (breaths per minute) for each condition in the IB task. 
Table 2 ANCOVA and Post hoc t-tests results for heart rate (bpm) and SDNN (ms)

\begin{tabular}{|c|c|c|c|c|c|c|c|c|c|c|c|c|c|}
\hline & \multicolumn{5}{|l|}{ ANCOVA } & \multicolumn{8}{|c|}{ Post hoc analysis } \\
\hline & effects & DF & $\mathrm{F}$ & $p$ & $\eta_{\mathrm{p}}^{2}$ & groups & $t$ & $p$ & $\begin{array}{c}p \\
\text { corr. }\end{array}$ & mean & SE & $\begin{array}{l}95 \% \text { CI } \\
\text { - lower }\end{array}$ & $\begin{array}{l}95 \% \mathrm{CI} \\
\text { - upper }\end{array}$ \\
\hline Heart & Feedback & 1 & 18.19 & .000 & 0.42 & FCF P-SCF P & -4.95 & .000 & .000 & -0.86 & 0.17 & -1.22 & -0.50 \\
\hline \multirow[t]{5}{*}{$\begin{array}{l}\text { rate } \\
(\mathrm{bpm})\end{array}$} & $\begin{array}{l}\text { Feedback } \\
* \text { IA } \\
\text { score }\end{array}$ & 1 & 0.20 & .656 & 0.01 & $\begin{array}{l}\text { FCF A-SCF } \\
\text { A }\end{array}$ & -2.29 & .03 & .12 & -0.39 & 0.17 & -0.74 & -0.04 \\
\hline & IB & 1 & 0.44 & .513 & 0.02 & FCF P-FCF A & -0.09 & .93 & 3.80 & -0.03 & 0.30 & -0.65 & 0.59 \\
\hline & $\begin{array}{l}\text { IB * IA } \\
\text { score }\end{array}$ & 1 & 0.14 & .715 & 0.01 & SCF P-SCF A & 1.299 & .21 & .92 & 0.44 & 0.34 & -0.26 & 1.14 \\
\hline & $\begin{array}{l}\text { Feedback } \\
* \text { IB }\end{array}$ & 1 & 6.30 & .019 & 0.20 & $\operatorname{dif}$ P-dif A & -2.49 & .02 & .08 & -0.47 & 0.19 & -0.86 & -0.08 \\
\hline & $\begin{array}{l}\text { Feedback } \\
* \text { IB * IA } \\
\text { score }\end{array}$ & 1 & 1.47 & .237 & 0.06 & & & & & & & & \\
\hline SDNN & feedback & 1 & 12.36 & .002 & 0.33 & FCF P-SCF P & -2.796 & .01 & .04 & -4.74 & 1.70 & -8.22 & -1.25 \\
\hline \multirow[t]{5}{*}{$(\mathrm{ms})$} & $\begin{array}{l}\text { Feedback } \\
* \text { IA } \\
\text { score }\end{array}$ & 1 & 0.10 & .75 & 0.004 & $\begin{array}{l}\text { FCF A-SCF } \\
\text { A }\end{array}$ & -3.13 & .004 & .016 & -5.07 & 1.62 & -8.40 & -1.74 \\
\hline & IB & 1 & 1.63 & .21 & 0.06 & FCF P-FCF A & 1.47 & .16 & .64 & 2.21 & 1.50 & -0.89 & 5.30 \\
\hline & $\begin{array}{l}\text { IB * IA } \\
\text { score }\end{array}$ & 1 & 0.05 & .82 & 0.002 & SCF P-SCF A & 0.89 & .38 & 1.52 & 1.87 & 2.10 & -2.44 & 6.18 \\
\hline & $\begin{array}{l}\text { Feedback } \\
* \text { IB }\end{array}$ & 1 & 0.03 & .86 & 0.001 & $\operatorname{dif}$ P-dif A & 0.18 & .86 & 3.44 & 0.33 & 1.90 & -3.50 & 4.20 \\
\hline & $\begin{array}{l}\text { Feedback } \\
* \text { IB * IA } \\
\text { score }\end{array}$ & 1 & 0.13 & .72 & 0.005 & & & & & & & & \\
\hline
\end{tabular}

Table 3 Wilcoxon signed-ranks test results for RMSSD ( $\mathrm{ms}$ ) and respiratory rate (bpm)

\begin{tabular}{llccrr}
\hline & Groups & $\mathrm{Z}$ & $p$ & \multicolumn{1}{c}{$\begin{array}{c}\mathrm{r} \\
\text { corrected }\end{array}$} \\
\hline RMSSD & SCF P-FCF P & -1.52 & 0.13 & 0.52 & -0.29 \\
& SCF A-FCF A & -0.74 & 0.46 & 1.84 & 0.14 \\
& FCF A-FCF P & -1.24 & 0.22 & 0.88 & -0.24 \\
& SCF A-SCF P & -0.66 & 0.51 & 2.04 & -0.13 \\
& dif Active-dif & -0.64 & 0.52 & 2.08 & 0.12 \\
\hline Respiratory rate & Passive & & & -0.12 \\
& SCF P-FCF P & -0.63 & 0.53 & 2.12 & 0.12 \\
& SCF A-FCF A & -0.60 & 0.55 & 2.20 & -0.23 \\
& FCF P-FCF A & -1.44 & 0.15 & 0.60 & -0.06 \\
& SCF P-SCF A & -0.34 & 0.74 & 2.96 & -0.21 \\
& dif Active-dif & -1.08 & 0.28 & 1.12 & \\
\hline
\end{tabular}


the active condition (FCF Active vs. SCF Active) $($ mean $=49.79, S D=14.52)$ was also significantly lower $[t(26)=-3.13, p=0.02$, Cohen's $d=$ $-0.60]$ than in the SCF blocks (mean $=54.86, S D$ $=14.72)($ Table 1 , Figure 1, Figure 2). There were no other significant results in paired $t$-tests (Table 2).

For the RMSSD, Wilcoxon tests showed no significant difference in HRV between conditions. The exact values are stated in Table 3. Spearman's correlation was run to determine the relationship between IA score and RMSSD in all conditions (FCF Passive, FCF Active, SCF Passive, SCF Active). There was no significant correlation between any condition and IA. Correlation coefficients did not exceed 0.29 and $p$ values were higher than 0.52 .

For the respiratory rate analysis, Wilcoxon tests showed no difference in respiratory rate between conditions. The exact values are stated in Table 3. Spearman's correlation was also run to determine the relationship between IA and ResR in all conditions (FCF Passive, FCF Active, SCF Passive, SCF Active). Correlation coefficients did not exceed 0.23 and $p$-values were higher than 0.6 in all conditions.

\section{Discussion}

This experiment investigated the changes in the HR, ResR and HRV measured as SDNN and RMSSD induced by FCF. The participants were exposed to alternating blocks of synchronous and accelerated false auditory cardiac feedback during two experimental tasks. The results demonstrated that false cardiac feedback affected the participants' HR, but in the opposite direction than hypothesized. There was a significant interaction between feedback and IB condition - the difference between FCF and SCF was greater in the passive condition than in the active condition. Although the HR was slower in the FCF blocks compared to the SCF blocks, the HRV measured as SDNN showed a conflict- ing result, being greater in the SCF blocks. This trend was not observed in HRV measured by RMSSD. Since RMSSD is an indicator of the short-term variability and SDNN reflects the overall variation within the RR interval series (Tarvainen et al., 2014), the results from these two analyses are not completely interchangeable. Interoceptive accuracy had no influence on any of the variables.

It was hypothesized that accelerated FCF would provoke an acceleration of HR and ResR and a decrease in HRV. Even though the HRV, measured as the SDNN, decreased in reaction to the FCF, the expected increase in the actual HR and ResR was not observed. In contrast, the FCF triggered a deceleration of the actual HR. After listening to the false heart beats, some participants reported that they tried to calm down when they noticed the change in the feedback. The aforementioned discrepancy between the hypothesis and the observed effect on HR could be due to the participants' successful attempt to down-regulate their HR during the FCF blocks.

There was a significant interaction between feedback and IB condition in HR. The HR was highest during synchronous feedback in the passive condition. This effect could be caused by varying attentional demands between the conditions of the task. The stimuli in the IB task were in auditory form, as well as the cardiac feedback and in the active condition, and also participants needed to pay more attention to the stimuli of the IB task than in the passive condition. Therefore, the effect of the cardiac feedback on the HR could have been mitigated in the active condition due to the separation of auditory attention.

Our results are contradictory to previous studies investigating the effect of FCF on physiological functions, which suggested that FCF had no effect on the participants' HR (Makkar \& Grisham, 2013) or on skin conductance (Kleint et al., 2015). In contrast, Story and Craske (2008) 
reported that healthy participants had a higher HR in the false-feedback blocks compared to the true-feedback blocks. There was no study with a similar effect to ours with FCF triggering a deceleration of HR.

There was no correlation between interoceptive accuracy and HR, ResR and HRV. This is in contrast with Füstös et al. (2012), who found that individuals with a better interoceptive ability were more successful in down-regulating their emotional response to negative affect. The discrepancy in our findings might be due to the difference in auditory stimuli, which might have triggered a smaller negative affect that failed to trigger this down-regulatory reaction.

Our experiment has several limitations. First, no baseline recordings for HR, ResR and HRV were acquired. Due to that, it was impossible to compare HR, ResR and HRV during the resting state with changes during the synchronous and the false feedback. Second, the sinus tone form of the feedback could have been perceived as less authentic by the participants. A study by Kleint et al. (2015) showed that participants reacted more to false feedback in the form of a heartbeat tone than to sinus tone stimulation. It is possible that a heartbeat form of auditory stimulation would have been perceived as more authentic and would have triggered different cardiac and respiratory changes. Third, participants' ability to discriminate between FCF and SCF was not explored systematically. Participants were questioned if they noticed changes in the feedback but more detailed questioning was not included in the debriefing. For that reason, the assumption that the observed decrease of heart rate during FCF is due to participants' effort to calm-down was based on information gathered during debriefing. Finally, our task could have been more challenging for the participants because both the false feedback and the stimuli used in the IB task were in an auditory modality.

\section{Conclusion}

The present study broadens findings about the possible effects of FCF on cardiac activity of healthy subjects. FCF elicited a change of HR in the opposite direction than was observed in previously published single study (Story \& Craske, 2008), possibly due to the successful downregulation of participants' heartbeat in reaction to accelerated cardiac feedback. Therefore, factors affecting participants' reaction to FCF should be considered in future studies, e.g. the levels of participant's awareness of cardiac feedback falsity or the negative affect associated with various auditory forms of cardiac feedback.

\section{References}

Barrett, L. F., \& Simmons, W. K. (2015). Interoceptive predictions in the brain. Nature Rewiews Neuroscience, 16(7), 419-429. doi: 10.1038/nrn3950

Berntson, G. G., Lozano, D. L., \& Chen, Y. J. (2005). Filter properties of root mean square successive difference (RMSSD) for heart rate. Psychophysiology, 42(2), 246-252. doi: 10.1111/j.1469-8986. 2005.00277.x

Bogaerts, K., Notebaert, K., Van Diest, I., Devriese, S., De Peuter, S., \& Van den Bergh, O. (2005). Accuracy of respiratory symptom perception in different affective contexts. Journal of Psychosomatic Research, 58(6), 537-543. doi: 10.1016/j. jpsychores.2004.12.005

Dimitriev, D. A., Dimitriev, A. D., Karpenko, Y. D., \& Saperova, E. V. (2008). Influence of examination stress and psychoemotional characteristics on the blood pressure and heart rate regulation in female students. Human Physiology, 34(5), 617-624. doi: 10.1134/S0362119708050101

Domschke, K., Stevens, S., Pfleiderer, B., \& Gerlach, A. L. (2010). Interoceptive sensitivity in anxiety and anxiety disorders: An overview and integration of neurobiological findings. Clinical Psychology Review, 30(1), 1-11. doi: 10.1016/j.cpr.2009.08.008 Füstös, J., Gramann, K., Herbert, B. M., \& Pollatos, O. (2012). On the embodiment of emotion regulation: Interoceptive awareness facilitates reappraisal. Social Cognitive and Affective Neuroscience, 8(8), 911917. doi: 10.1093/scan/nss089 
Garfinkel, S. N., Seth, A. K., Barrett, A. B., Suzuki, K., \& Critchley, H. D. (2015). Knowing your own heart: Distinguishing interoceptive accuracy from interoceptive awareness. Biological Psychology, 104, 6574. doi: 10.1016/j.biopsycho.2014.11.004

Gray, M. A., Harrison, N. A., Wiens, S., \& Critchley, H. D. (2007). Modulation of emotional appraisal by false physiological feedback during fMRI. PLoS one, 2(6), e546. doi: 10.1371/journal.pone.0000546

Kever, A., Pollatos, O., Vermeulen, N., \& Grynberg, D. (2015). Interoceptive sensitivity facilitates both antecedent- and response-focused emotion regulation strategies. Personality and Individual Differences, 87, 20-23. doi: 10.1016/j.paid.2015.07.014

Kleint, N. I., Wittchen, H. U., \& Lueken, U. (2015). Probing the interoceptive network by listening to heartbeats: An fMRI study. PloS one, 10(7), e0133164. doi: 10.1371/journal.pone.0133164

Makkar, S. R., \& Grisham, J. R. (2013). Effects of false feedback on affect, cognition, behavior, and postevent processing: The mediating role of selffocused attention. Behavior Therapy, 44(1), 111124. doi: 10.1016/j.beth.2012.07.005

Murphy, J., Brewer, R., Catmur, C., \& Bird, G. (2017) Interoception and psychopathology: A developmental neuroscience perspective. Developmental Cognitive Neuroscience, 23, 45-56. doi: 10.1016/ j.den.2016.12.006

Nejedly, P., \& Virgala, J. (2016). Physiocrate: A SignalPlant Toolbox for Respiratory, Blood Pressure and EMG Signal Analysis. Student EEICT, pp. 53-55. doi: 10.1088/0967-3334/37/7/N38

Pollatos, O., Kurz, A. L., Albrecht, J., Schreder, T. Kleemann, A. M., Schöpf, V., Kopietz, R., Wiesmann, M., \& Schandry, R. (2008). Reduced perception of bodily signals in anorexia nervosa. Eating Behaviors, 9(4), 381-388. doi: 10.1016/j.eatbeh.2008. 02.001

Pollatos, O., Traut-Mattausch, E., Schroeder, H., \& Schandry, R. (2007). Interoceptive awareness mediates the relationship between anxiety and the intensity of unpleasant feelings. Journal of Anxiety Disorders, 21(7), 931-943. doi: 10.1016/j.janxdis. 2006.12 .004

Rieber, N., Betz, L., Enck, P., Muth, E., Nikendei, C., Schrauth, M., Werner, A., Kowalski, A., \& Zipfel, S. (2009). Effects of medical training scenarios on heart rate variability and motivation in students and simulated patients. Medical Education, 43(6), 553-556. doi: $10.1111 / \mathrm{j} .1365-2923.2009 .03374 . \mathrm{x}$
Seth, A. K. (2013). Interoceptive inference, emotion, and the embodied self. Trends in Cognitive Sciences, 17(11), 565-573. doi: 10.1016/j.tics.2013.09.007

Seth, A. K., Suzuki, K., \& Critchley, H. D. (2012). An interoceptive predictive coding model of conscious presence. Frontiers in Psychology, 2(395). doi: 10.3389/fpsyg.2011.00395

Schandry, R. (1981). Heart beat perception and emotional experience. Psychophysiology, 18(4), 483488. doi: 10.1111/j.1469-8986.1981.tb02486.x

Stevens, S., Gerlach, A. L., Cludius, B., Silkens, A., Craske, M. G., \& Hermann, C. (2011). Heartbeat perception in social anxiety before and during speech anticipation. Behaviour Research and Therapy, 49(2), 138-143. doi: 10.1016/j.brat.2010.11.009

Story, T. J., \& Craske, M. G. (2008). Responses to false physiological feedback in individuals with panic attacks and elevated anxiety sensitivity. Behaviour Research and Therapy, 46(9), 1001-1008. doi: 10.1016/j.brat.2008.06.001

Tarvainen, M. P., Niskanen, J. P., Lipponen, J. A., Ranta-Aho, P. O., \& Karjalainen, P. A. (2014). Kubios HRV - heart rate variability analysis software. Computer Methods and Programs in Biomedicine, 113(1), 210-220. doi: 10.1016/j.cmpb.2013.07.024

Van der Does, A. J. W., Antony, M. M., Ehlers, A., \& Barsky, A. J. (2000). Heartbeat perception in panic disorder: A reanalysis. Behaviour Research and Therapy, 38(1), 47-62. https://www.researchgate.net/ publication/38306318_Heartbeat_perception_ in_panic_disorder_A_reanalysis

Wiens, S. (2005). Interoception in emotional experience. Current Opinion in Neurology, 18(4), 442447. doi: 10.1097/01.wco.0000168079.92106.99

Wiens, S., Mezzacappa, E. S., \& Katkin, E. S. (2000). Heartbeat detection and the experience of emotions. Cognition \& Emotion, 14(3), 417-427. doi: 10.1080/026999300378905

Wild, J., Clark, D. M., Ehlers, A., \& McManus, F. (2008). Perception of arousal in social anxiety: Effects of false feedback during a social interaction. Journal of Behavior Therapy and Experimental Psychiatry, 39(2), 102-116. doi: 10.1016/j.jbtep.2006. 11.003

Yoris, A., Esteves, S., Couto, B., Melloni, M., Kichic, R., Cetkovich, M., Favaloro, R., Moser, J., Manes, F., Ibanez, A., \& Sedeño, L. (2015). The roles of interoceptive sensitivity and metacognitive interoception in panic. Behavioral and Brain Functions, 11(1), 1-6. doi: 10.1186/s12993-015-0058-8 